\title{
Hybrid silicon lasers for optical interconnect
}

\author{
(Invited Paper) \\ Y. De Koninck, S. Keyvaninia, S. Stankovic, D. Van Thourhout, G. Roelkens and R. Baets \\ Department of Information Technology, Photonics Research Group, Ghent University - IMEC \\ Center for Nano- and Biophotonics (NB-Photonics) \\ Sint-Pietersnieuwstraat 41 B-9000 Ghent, Belgium \\ email: yannick.dekoninck@intec.ugent.be
}

\begin{abstract}
Hybrid III-V/silicon lasers for short reach optical interconnect applications need to have a modest power dissipation and preferably also a small footprint. Furthermore they should be free of mode hops even under varying temperature conditions. In this paper a number of designs will be discussed that address this set of requirements.

Index Terms-Silicon photonics, heterogeneous integration, hybrid lasers, optical interconnects
\end{abstract}

\section{INTRODUCTION}

As the number of microprocessor cores that are integrated on a single die increases rapidly, the amount of data that has to be delivered to and extracted from the chip scales accordingly. If this trend continues in the coming decade, electrical interconnects will no longer be able to deliver the required bandwidth in a cost- and power-effective way and optical interconnects will be needed both for on- and off-chip communication in future generation integrated circuits [1]. To offer sufficient advantage over electrical wiring, the power budget for such short-range, high-speed communication systems needs to be a few $100 \mathrm{fJ} /$ bit for offchip interconnects and even lower for on-chip communication. Only a fraction of the available power can be allocated to the transmitter circuit, consisting of the laser and/or modulator and its drive circuitry. To reach such ambitious goals, it is obvious that an efficient, low-threshold laser source is necessary. Furthermore, wavelength division multiplexing is put forward to increase the effective bandwidth of the system, but this also puts stringent requirements on the spectral properties of the laser source: single mode operation is essential and instabilities such as temperature variation induced mode-hops are not allowed. Mode hops should also be avoided in single channel systems so as to avoid bit errors [2].

To solve this problem, a number of solutions have been proposed. Externally generated laser light can be fed into the circuit using a fiber or a laser source can be flip-chipped directly onto the chip. However, the highest degree of efficiency and the smallest footprint can potentially be reached by heterogeneously integrating the laser sources onto the photonic/electronic IC [3]. In this paper we will review a number of designs for such lasers that are suitable for optical interconnects. We will focus on approaches that have a wavelength selection mechanism defined in

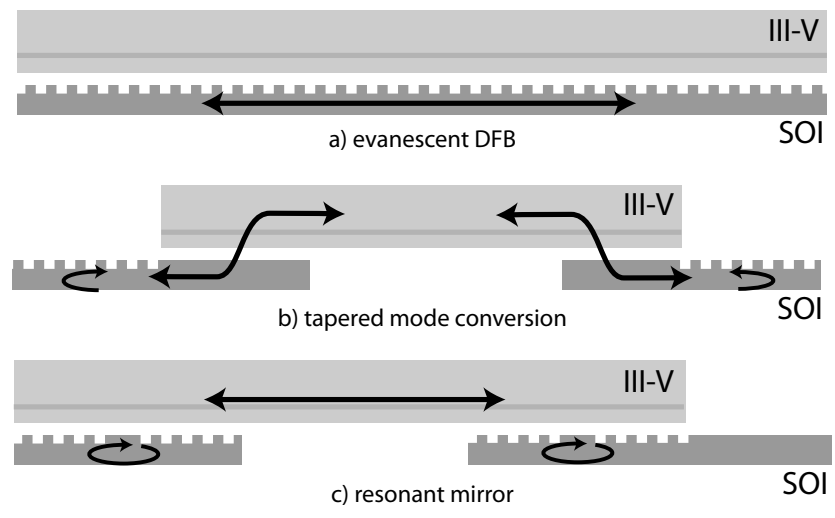

Fig. 1. Schematic overview of the operation of the evanescent DFB laser (a), III-V-confined laser with adiabatic mode-conversion (b) and resonant mirror laser (c)

the silicon layer to avoid multi-mode operation or modehopping. Although we only discuss this type of lasers in the context of optical interconnect, an array of single-mode lasers, each operating at a well defined wavelength can also be used as a tunable light source in spectroscopic applications.

Perhaps the most straight-forward approach is the silicon evanescent laser. The laser consists of an optical waveguide in the silicon layer and a wide III-V mesa bonded on top. The laser mode is mainly confined to the silicon waveguide and only its evanescent tail overlaps with the quantum wells in the III-V layer. DFB-lasers are fabricated by etching periodic corrugations in the silicon wire to obtain distributed feedback. Given the excellent spectral properties found in DFB lasers and the high-resolution fabrication technology available for silicon photonics, evanescent DFB lasers can easily be used in a wavelength division multiplexing scheme to boost the effective bandwidth through a single output channel. This approach was demonstrated for both direct [4] and adhesive bonding [5]. Due to the evanescent nature of the gain mechanism, the modal gain is limited, typically leading to a lasing threshold for evanescent DFB lasers of about 20 $\mathrm{mA}$ and a slope efficiency of $0.067 \mathrm{~W} / \mathrm{A}$, with a maximum output power of about $5 \mathrm{~mW}$ at $100 \mathrm{~mA}$ driving current [4]. Assuming typical values for the required optical power for 
error-free operation, this leads to an energy consumption of the order of $1 \mathrm{pJ} / \mathrm{bit}$, which is far above the goals defined above. Splitting the power into different spatially separated channels, each individually modulated, can alleviate this issue, but still, reaching the $100 \mathrm{fj} / \mathrm{bit}$ goal is very ambitious.

\section{Resonant MirRor LASERS}

In order to reduce the power consumption of the laser, the overlap of the optical field with the III-V multiple quantum well region should be optimized and the laser cavity volume should be minimized. However, still the wavelength selective feedback is preferably implemented in the silicon waveguide layer, given the fact that high accuracy patterning is more easily achieved in silicon than in III-V layers. These requirements can be realized by defining a waveguide in the III-V layer which improves the confinement in the active region. In order to couple the light to the silicon waveguide layer, typically an intra-cavity spot-size converter is used to squeeze the mode out of the III-V mesa into the silicon waveguide. In the silicon waveguide, wavelength selective feedback can be realized by using a combination of Bragg gratings and ring resonators, which can be thermo-optically tuned [6]. However, low-loss and low-back reflection tapers are difficult to realize in such a device geometry, which again impacts the threshold current and slope efficiency of the device.

Recently, we proposed a novel approach for realizing low-power consumption, single mode lasers that are robust against mode hops based on resonant mirrors [7]. Still the laser mode is confined to the III-V wire but instead of adiabatically tapering all the light from the III-V wire to the silicon layer towards the wavelength selective feedback structure, the III-V waveguide is now evanescently coupled to two silicon cavities, one at each facet of the III-V wire. The silicon cavities are identical and the coupling to the III-V waveguide provides high, narrow banded reflectivity back into that III-V waveguide as described in [7]. Figure 2 shows a typical reflection and transmission spectrum of a single silicon cavity coupled to a III-V waveguide.

The reflectivity of this mirror is mainly determined by the quality of the silicon cavity. One can prove that if the losses in the cavity are sufficiently small, this mirror will reflect near $100 \%$ of the incident light back into the III-V waveguide. This is similar to an add-drop filter, which is always critically coupled if the coupling section is symmetric and the losses in the ring are sufficiently low.

Typically, the silicon cavities are 20-30 $\mu \mathrm{m}$ long, and because they provide such high reflectivity, the laser gain section can also be short, on the order of a few 10's of micrometers. This means that the device length can be in the range of $50-100 \mu \mathrm{m}$, which is significantly shorter than the approaches presented earlier. The threshold current should decrease proportionally, making this type of laser a viable candidate

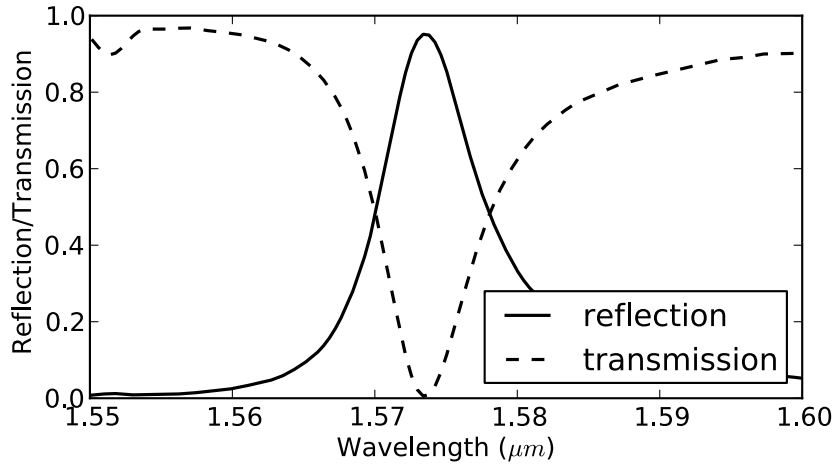

Fig. 2. Typical simulated characteristic of a resonant mirror. This mirror is about $30 \mu \mathrm{m}$ long and reflects $95 \%$ of the incident light (FWHM $8 \mathrm{~nm}$ ).

as a light source in future optical interconnects.

The reflection bandwidth of the resonant mirror is determined by the coupling between the III-V wire and the silicon cavity. Decreasing the coupling will narrow the reflection band-width and vice-versa. This way the reflection bandwidth can be engineered to reflect only one longitudinal laser mode. If the cavity is sufficiently short it can be designed to oscillate at a frequency aligned to the mirror reflection peak and to do so over a substantial temperature range.

\section{CONClusions}

Hybrid silicon lasers based on bonded III-V layers on silicon were discussed with respect to the challenges and trade-offs in their design and fabrication. Focus is on compact designs that combine good light confinement in the gain layer with good spectral control provided by grating structures patterned in silicon.

\section{ACKNOWLEDGEMENTS}

The authors acknowledge partial support from IWT through the SBO-Glucosens project as well as from ERC through the InSpectra project. Yannick De Koninck thanks the research foundation Flanders (FWO) for a research grant.

\section{REFERENCES}

[1] D. A. B. Miller, "Device Requirements for Optical Interconnects to Silicon Chips," Proceedings of the IEEE, vol. 97, no. 7, 2009.

[2] T. Heumier and J. Carlsten, "Detecting mode hopping in semiconductor lasers by monitoring intensity noise," IEEE Journal of Quantum Electronics, vol. 29, no. 11, pp. 2756-2761, 1993.

[3] G. Roelkens, L. Liu, D. Liang, R. Jones, A. Fang, B. Koch, and J. Bowers, "III-V/silicon photonics for on-chip and inter-chip optical interconnects," Laser \& Photonics Reviews, vol. 4, no. 6, pp. 751-779, Nov. 2010.

[4] A. W. Fang, E. Lively, H. Kuo, D. Liang, and J. E. Bowers, "A distributed feedback silicon evanescent laser," Optics Express, vol. 16, no. 7, pp. 4413-4419, Mar. 2008.

[5] S. Stankovic, G. Roelkens, and D. Van Thourhout, "In preparation."

[6] G.-H. Duan, C. Jany, A. Le Liepvre, J. Provost, D. Make, F. Lelarge, M. Lamponi, F. Poingt, J. Fedeli, S. Messaoudene, D. Bordel, S. Brision, S. Keyvaninia, G. Roelkens, and D. Van Thourhout, "10 Gb/s integrated tunable hybrid III-V/Si laser and silicon MZ modulator," in ECOC, 2012.

[7] Y. De Koninck, G. Roelkens, and R. Baets, "Cavity Enhanced Reflector Based Hybrid Silicon Laser," in IEEE Photonics Society Annual Meeting 2010, 2010. 